\title{
Red colorants from filamentous fungi: Are they ready for the food industry?
}

4 Laurent Dufossé , $^{1,2}$

$5 \quad{ }^{1}$ Laurent Dufossé, Laboratoire de Chimie des Substances Naturelles et des Sciences des

6 Aliments, ESIROI Agroalimentaire, Université de La Réunion, F-97490 Sainte-Clotilde, Ile

7 de La Réunion, France

$8 \quad{ }^{2}$ Laboratoire ANTiOX, Université de Bretagne Occidentale, Pôle Universitaire Pierre-Jakez

9 Hélias, Quimper, France

10 Correspondence: laurent.dufosse@univ-reunion.fr (L. Dufossé)

11 Abstract

12 Food components of microbial-origin have a long history in food science and the food

13 industry. Thickening and gelling agents, flavour enhancers, polyunsaturated fatty acids,

14 flavour compounds, vitamins, essential amino acids, and acidulants are some examples of

15 such ingredients. This paper will provide an update on the current worldwide situation for

16 four different fungal reds: (i) carotenoid lycopene (simple compound, complex current status);

17 (ii) molecular biology on Monascus to avoid mycotoxin and cholesterol-lowering substance in

18 pigmented extracts; (iii) newcomers with azaphilone-producing fungi such as Talaromyces

19 atroroseus, Penicillium purpurogenum, and Talaromyces albobiverticillius; and (iv)

20 anthraquinones as a possible alternative to the insect-sourced carmine. The future of 
Monascus in Europe and the USA is just around the corner, and markets will appear as soon as the citrinin issue has been solved, with the help of the current better knowledge of full genomes from industrial strains. Fungi bring a new class of pigments to the food industry, as azaphilones are not present in plants. These azaphilone-producing strains should now be thoroughly studied through liquid fermentation of Penicillium/Talaromyces strains, with optimized scale-up. A fungal alternative to carmine insect anthraquinone is further away from the market, however, due to the particular stability of this vibrant red in foods; research efforts should be intensified.

Keywords: Red pigments; Food ingredient; Colorant; Carotenoid; Lycopene; Azaphilone; Anthraquinone; Blakeslea; Monascus; Talaromyces

\section{You're already eating microbial metabolites all day long}

Ingredients derived from microbial fermentation are steadily gaining ground in the food industries. Thickening or gelling agents (e.g. polysaccharides such as xanthan, curdlan, gellan), flavour enhancers (yeast hydrolysate, monosodium glutamate), polyunsaturated fatty acids (PUFAs), flavour compounds (gamma-decalactone, diacetyl, methyl-ketones), vitamins, essential amino acids, and acidulants (lactic acid, citric acid) are some examples illustrating this trend (Mc Neil et al., 2013). Efforts have been made, and are continuing, to reduce the production costs of pigments produced by microbial fermentation, since synthetic pigments or those extracted from natural plant sources can often be produced more economically (Dufossé, 2008; Dufossé, 2017a). The successful marketing of natural pigments such as $\beta$ carotene, lutein, and astaxanthin derived from microalgae (i.e. non-conventional sources) or extracted from plants (conventional sources), both as food colorants and nutritional supplements, reflects the presence and importance of niche markets in which consumers are willing to pay a premium for 'natural healthy ingredients'. 
Among other non-conventional sources, filamentous fungi are known to produce an extraordinary range of pigments that include several chemical classes such as carotenoids, melanins, azaphilones, anthraquinones, flavins, phenazines, quinones, and more specifically, violacein and indigo (Dufossé, 2008). The success of any class of pigment produced by fermentation depends on its acceptance by the consumers, regulatory approval, and the capital investment required bringing the product onto the market. Twenty years ago, influential representatives from the food industry expressed doubts about the successful commercialization of algae-derived and fermented food grade pigments due to the high investment required for open ponds, photo-bioreactors and fermentation facilities, and the extensive and lengthy toxicity studies required by the regulatory authorities. Poor public perception of fungal-derived products for food use also had to be taken into account (Milićević et al., 2010). Nowadays, some fungal food grade pigments obtained by fermentation already exist on the market worldwide. Among them, fungal Monascus pigments, Arpink red ${ }^{\mathrm{TM}}$ (now Natural Red ${ }^{\mathrm{TM}}$ ) produced by Penicillium oxalicum, riboflavin from the mould fungus Ashbya gossypii, lycopene and $\beta$-carotene from the tropical mold Blakeslea trispora. As an example, the production yield of $\beta$-carotene may be as high as 17g/L of the Blakeslea trispora culture medium (Dufossé, 2016; Torres et al., 2016).

The present opinion paper gives an update about the worldwide current situation for some fungal reds chosen for the history of their long use in Asia or Europe, the diversity of their chemical structures, and the strategy of the development from lab research to the market (see Fig. 1):

- Carotenoid lycopene (simple compound, long-standing history, complex current status)

- Molecular biology on Monascus to avoid mycotoxin and cholesterol-lowering substance in pigmented azaphilone extracts, 
- Newcomers with azaphilone-producing fungi such as Talaromyces atroroseus, Penicillium purpurogenum, Penicillium marneffei, Talaromyces albobiverticillius

- Focus on anthraquinones.

To sum up, those who are still afraid of fungal products in food and feed, please have a look inside your pigmented plant extracts! Are you sure they are mycotoxin free (Solfrizzo et al., 2015)?

\section{Fungal red carotenoid already produced on a large scale: lycopene from Blakes/ea trispora}

In the European Union, the United States of America (USA), Canada, Australia and New Zealand, pioneering work on large-scale production of fungal colorants has been done on carotenoids. Academics knew for a long time that fungi belonging to the order Mucorales were able to produce $\beta$-carotene (Ciegler, 1965). The first papers dealing with Blakeslea trispora carotenoid production were published in the late 1950s (Ciegler et al., 1959; Ciegler, 1965). It took four decades to move to industrial production, waiting for consumer interest in natural colorants, and to develop biotechnological techniques. The first fungal carotenoid launched in Western Europe was $\beta$-carotene between 1995 and 2001 (European Commission directive $N^{\circ}$ 50/2001), by the Dutch company Gist Brocades (now DSM). Soviet Union companies had already been doing the same thing in Eastern Europe a decade earlier. As lycopene is a metabolic intermediate in the biosynthesis of $\beta$-carotene, the use of inhibitors opens the doors for its industrial production, together with the use of lycopene-accumulating and overproducing mutants. Vitatene, a Spanish company, filed a novel foods and novel food ingredients application in 2003, to place lycopene from Blakeslea trispora on the European 
market (under Regulation EC $\mathrm{N}^{\circ} 258 / 97$ ). A positive response was published on 23 October 2006 (European Commission decision N 721/2006).

On the market, this biotech colorant has to compete with lycopene extracted from tomato (E160d(ii), listed in European directive 94/36/EC) and with the cost-effective synthetic lycopene (E160d(i)), from chemical synthesis. As a result of the increased demand of consumers for natural and safe food ingredients, the toxicological aspects of biotech lycopene E160d(iii) was investigated within the framework of a ninety-day oral toxicity study in rats (Jonker et al., 2003). The results from this study do not provide any evidence of toxicity for lycopene extracted from the biomass of Blakeslea trispora at dietary levels up to $1.0 \%(\mathrm{w} / \mathrm{w}$, as a suspension in sunflower oil) as demonstrated by the findings of clinical observations, neurobehavioral observations, motor activity assessment, body weight and food consumption measurements, ophthalmoscopic examinations, hematology, clinical chemistry, urinalysis, organ weights, gross pathology, or histopathology. The No-Observed-Effect Level (NOEL) was $1.0 \%(\mathrm{w} / \mathrm{w})$ in the diet, the highest dietary concentration tested (Jonker et al., 2003).

Lycopene has received particular attention in recent years as a result of studies that have reported that it is a highly efficient antioxidant and has a high singlet-oxygen and free-radical scavenging capacity. Many researchers have shown that lycopene is detected in the plasma and tissues of humans following incorporation into the daily diet. Such studies indicate that lycopene is absorbed and subsequently distributed to the tissues. The natural lycopene sales started on this health market, on functional foods.

Average dietary intakes of lycopene from foods in different populations are, according to dietary surveys, estimated to be between 0.5 and $5 \mathrm{mg} /$ day, with high intakes up to about 8 $\mathrm{mg} /$ day. High intakes of fruit and vegetables, especially tomato products, may result in occasional intakes of $20 \mathrm{mg} /$ day or more. 
In 2005 Vitatene company informed the European Food Safety Authority (EFSA) that use

119 levels of lycopene from B. trispora in foodstuffs would lead to an additional intake of up to

120 about $2 \mathrm{mg} /$ day. The proposed use level of lycopene in food supplements would give rise to

121 an additional intake of $20 \mathrm{mg} /$ day. To date, no long-term feeding studies conducted with

122 lycopene extracted from the microorganism B. trispora have been performed. The

123 toxicological data on $\alpha$-tocopherol containing oil suspensions of lycopene from B. trispora

124 (90-day oral feeding study) are not sufficient to derive an acceptable daily intake (ADI).

125 EFSA concluded at that time that $\alpha$-tocopherol-containing an oil suspension of lycopene,

126 obtained from B. trispora, for use as a novel food ingredient in foodstuffs leading to an

127 additional intake of up to about $2 \mathrm{mg}$ /day was not of concern from the safety point of view.

128 However, this does not hold for the proposed levels of use of lycopene in foods that would

129 give rise to an additional intake of $20 \mathrm{mg}$ per day.

130 The true use of lycopene as a food colorant is a more complex situation. In Europe, EFSA

131 currently allows this use within the framework of an Acceptable Daily Intake (ADI) of 0.5

$132 \mathrm{mg} / \mathrm{kg}$ body weight (bw)/day based on No-Observed-Adverse-Effect Level (NOAEL)

133 published data. In 2009 the FAO/WHO Joint Expert Committee on Food Additives (JECFA)

134 replaced the group ADI of $0-0.5 \mathrm{mg} / \mathrm{kg}$ bw with a group ADI 'not specified' for lycopene

135 from all sources, creating a divergence (EFSA, 2010). Then, in the USA the Food and Drug

136 Administration approved a petition from LycoRed company seeking the green light to use

137 higher levels of tomato lycopene to restore colour to processed meats, giving manufacturers

138 of sausages, deli meats and jerky an alternative to synthetic FD\&C Red \#40 (Allura Red AC,

139 EC 129) and 'bug-derived' carmine (Watson, 2014). The present situation will continue to

140 evolve and it is possible that biotech lycopene will be clearly allowed in the next

141 months/years as a true colour in the food industry. 
142 To conclude this overview, it is noteworthy to mention that the development of biotech

143 lycopene took decades (Dufossé, 2017b; Mantzouridou and Tsimidou, 2008) and regulatory

144 aspects continue to affect its use. Such an impressive complexity with this well-known

145 carotenoid is just a small 'sneak peek' at the huge efforts yet to be accomplished with other

146 pigmented molecules such as azaphilones and anthraquinones. However, as for some

147 suppliers, lycopene red is 'more of an orangish-red, and not a true, vibrant red shade', and is

148 'also one of the more expensive natural colour options to use', the scientific community must 149 continue to investigate for natural reds.

\section{Towards a safe use of Monascus}

151 Monascus has been used to produce natural colorants and food supplements for more than one

152 thousand years in Asia, and more than one billion Asian people consume Monascus-

153 fermented products with their daily diet. The first known source reporting the use of these red

154 colorants was a recipe for the preparation of red pot-roast lamb, in which meat was simmered

155 with hong qu (red rice koji, made with Monascus purpureus), as handed down to Qing Yilu in

156 CE 965. Monascus species are known to produce six major azaphilone pigments, namely the

157 yellow monascin and ankaflavin, the orange monascorubrin and rubropunctatin, and the red

158 monascorubramine and rubropunctamine. To date, more than 50 different chemical structures

159 have been identified (Yang et al., 2015), because azaphilones easily combine with nitrogen-

160 containing compounds. Using next-generation sequencing and optical mapping approaches, a

161 24.1-Mb complete genome of a Monascus purpureus YY-1 industrial strain has been

162 described for the first time, and this will allow huge improvements in the process in the

163 coming years (Yang et al., 2015). It consists of eight chromosomes and 7491 genes. $M$.

164 purpureus should belong to the Aspergillaceae, mainly comprising the genera Monascus, 
Penicillium, and Aspergillus. Phylogenetic analysis at the genome level provides the first comprehensive prediction of the biosynthetic pathway for Monascus pigments.

167 Comparative genomic analyses demonstrated that the genome of $M$. purpureus is $13.6-40 \%$ smaller than that of closely related filamentous fungi and has undergone significant gene losses, most of which likely occurred during its specialized adaptation to starch-based foods.

170 Some polyketide synthases (PKS) are expressed at high levels under high-pigment-yielding

171 conditions. The citrinin PKS C6.123 has also been found in the genome (Yang et al., 2015),

172 paving the way for research aiming at non-mycotoxin producing strains, if suppression of the

173 citrinin gene does not change the ability of the strain to produce pigments, which seems to be

174 feasible, as described by Fu et al. (2007), who have shown that monascorubrin and citrinin are

175 synthesized by two separate pathways, because when the PKS gene responsible for synthesis

176 of citrinin was disrupted, red pigment production from the fungus was not affected.

177 Comparative transcriptome analysis revealed that carbon starvation stress, resulting from the 178 use of relatively low-quality carbon sources, contributed to the high yield of pigments by 179 suppressing central carbon metabolism and augmenting the acetyl-CoA pool. As for other 180 pigments produced by biotechnology, the problem is to have enough carbon oriented in the 181 correct pathway, i.e. the pigment pathway.

\section{Monascus-like pigments (MLPs) produced by Penicillium/Talaromyces species}

184 Some species of Talaromyces (the teleomorphic (sexual) stage of Penicillium) secrete large

185 amounts of red pigments. In the literature, this biosynthetic potential has been linked to

186 species such as Talaromyces purpurogenus, T. albobiverticillius, T. marneffei, and T.

187 minioluteus, often known under their previous Penicillium names (e.g. Penicillium sp. from 
Japan, Ogihara et al., 2000). However, some of them do not exert enough stability for pigment production, and should then be avoided for scaled-up production (Figure 2).

Woo et al. (2014) from Hong Kong investigated another filamentous fungus, Penicillium (Talaromyces) marneffei, for production of azaphilones exhibiting black, yellow and red hues.

The polyketide gene cluster and biosynthetic pathway were reported for monascorubrin in this red pigment-producing, thermal dimorphic fungus, taking advantage of available genome sequence and faster growth rate compared to Monascus species (Woo et al., 2014). The red pigment of $P$. marneffe $i$ has been shown to consist of a mixture of more than 16 chemical compounds, which are amino acid conjugates of monascorubrin and rubropunctatin, as amino acids can be conjugated under specific conditions without enzymatic catalysis, i.e. by Schiff base formation (Woo et al., 2014).

The aforementioned polyketide gene cluster and pathway have also been shown to be responsible for the biosynthesis of ankaflavin and citrinin, the latter being a mycotoxin exerting nephrotoxic activity in mammals (Kumar et al., 2014). Twenty-three putative PKS genes and two putative PKS-non-ribosomal peptide synthase hybrid genes were identified in the P. marneffei genome (Woo et al., 2014). Woo et al. (2014) systematically knocked out all 25 PKS genes of P. marneffei. They also knocked out genes located up- and downstream of the PKS gene responsible for red pigment production, and characterized the pathway for biosynthesis of the red pigment. However, it is still questionable whether it will be possible to produce mevinolin/lovastatin-free (a cholesterol-lowering drug that is undesirable in normal foods) and citrinin-free red pigments from P. marneffei, as the latter, a mycotoxin, appears to be an early byproduct of the biosynthetic pathway.

Isolates identified as T. purpurogenus have been reported to be of industrial interest (some of which are investigated in Mexico, Morales-Oyervides et al., 2015). They can produce 
212 extracellular enzymes and red pigments, but may also produce mycotoxins such as rubratoxin

213 A and B and luteoskyrin in addition to extrolites that may be toxic following intraperitoneal

214 (spiculisporic acid) and intravenal (rugulovasine A and B) injections in cats (Frisvad et al.,

215 2004). Consequently, mycotoxin production may limit the use of isolates of a particular

216 species in biotechnology, and Frisvad et al. (2013) concluded that Talaromyces purpurogenus

217 may thus not be recommended for industrial production of red pigments. Talaromyces

218 atroroseus sp. nov., described by the same group from Denmark, produces the azaphilone

219 biosynthetic families mitorubrins and Monascus-like pigments without being accompanied by

220 mycotoxin synthesis (patent applications WO2012022765 Mapari et al., 2012,

221 US20110250656 Mapari et al., 2011). As it has been found for Monascus, these azaphilone

222 pigments may react with amino groups containing compounds, to which reaction they owe

223 their name, providing intense dark red colours (Mapari et al., 2010; Gao et al., 2013).

224 A strain of Talaromyces albobiverticillius isolated in the lagoon from Réunion Island, Indian

225 Ocean, is currently being developed and optimized for red azaphilones production (please

226 refer to the two papers from this Pigments in Food congress, Journal of Food Composition

227 and Analysis, current special issue: Fouillaud M. et al., Production of pigments from the

228 tropical marine-derived fungus Talaromyces albobiverticillius: new resources for red natural

229 coloured metabolites; Venkatachalam M., Partial characterization of the pigments produced

230 by the marine-derived fungus Talaromyces albobiverticillius 30548 . Towards a new fungal

231 red colorant for the food industry).

232 insert Figure 2

\section{The anthraquinone quest}

234 Anthraquinones are widely spread throughout the kingdom of fungi (Caro et al., 2016), and

235 thus, the latter might serve as alternative sources since they are independent of agro-climatic 
conditions, in contrast to plant- and animal-derived sources. For example, anthraquinones

237 were found in Aspergillus sp., Eurotium sp., Fusarium sp., Dreschlera sp., Penicillium sp.,

238 Emericella purpurea, Curvularia lunata, Mycosphaerella rubella, Microsporum sp., etc.

239 (Caro et al., 2012; Gessler et al., 2013).

240 Anthraquinones exhibit a broad range of biological activities, including bacteriostatic,

241 fungicidal, antiviral, herbicidal, and insecticidal effects (Gessler et al., 2013). Presumably, in

242 fungi, these compounds are involved in interspecific interactions. For example,

243 anthraquinones synthesized by endophytic fungi protect the host plant from insects or other

244 microorganisms (Gessler et al., 2013).

245 The present picture of fungal anthraquinones is quite complex, with a great variety of

246 chemical structures, a huge number of factors or parameters which may have an effect on the

247 composition of quinoidal pigments biosynthesized by a particular species (Fouillaud et al.,

248 2016). Among them, e.g. habitat, light, $\mathrm{pH}$, temperature, $\mathrm{O}_{2}$ transfer, liquid/solid media,

249 culture medium, $\mathrm{C}$ and $\mathrm{N}$ sources, $\mathrm{C}: \mathrm{N}$ ratio, presence of organic acids, mineral salts, and

250 inoculum have been considered (Caro et al., 2012).

251 Today, research places the priority on a small number of fungal anthraquinone-producing

252 species meeting the following profile of requirements established by Mapari et al. (2009)

253 during the identification of potentially safe fungal cell factories for the production of

254 polyketide natural food colorants using chemotaxonomic rationale:

- the fungus shall be non-pathogenic to humans;

- the fungus shall be non-toxigenic under a broad range of production conditions; and

- the fungus shall be able to be produced in liquid media. 


\section{Plant-pigment preparations may contain fungal metabolites}

259 For most people, plants for food use appear safe, and they appear as sources of pristine

260 ingredients. This is not always true, and we mention here a grape byproduct, namely grape

261 pomaces, as an example. Grape pomaces are used as plant food supplements and food

262 colourings (anthocyanins). Unfortunately, these grape pomaces can be contaminated by 263 ochratoxin A (OTA), a mycotoxin produced mainly by Aspergillus carbonarius on grape

264 berries on the vine. OTA possesses nephrotoxic, immunosuppressive, teratogenic, and 265 carcinogenic properties.

266 Levels of contamination are presented in a nice paper from Solfrizzo et al. (2015). OTA was 267 found in $69 \%$ of food colouring samples at levels between 1.16 and $32.00 \mu \mathrm{g} / \mathrm{kg}$. The 268 situation appear so bad (high incidence of OTA contamination) that the authors recommend to 269 established maximum permitted levels for this mycotoxin in food colouring agents derived 270 from Vitis vinifera.

271 There is also much awareness in the wine industry where OTA decontamination of musts and 272 wines is under study, using inactivated yeasts or yeast cell walls (Petruzzi et al., 2015). Since 273 vintage year 2006, with the adoption of Regulation CE123/05, the level of OTA in 274 commercial wines cannot exceed $2 \mu \mathrm{g} / \mathrm{L}$, but many trade agreements usually require lower 275 limits (e.g. $0.5 \mu \mathrm{g} / \mathrm{L})$.

\section{Conclusion: Simple messages I wanted to deliver}

277 These four examples of fungal reds for the food industry describe diverse situations, from

278 products already on the market (lycopene from Blakeslea, red rice from Monascus) to

279 products still under development (azaphilones from Penicillium/Talaromyces, anthraquinones). The future of Monascus in Europe and the USA exists, and markets will 
281

282

283

284

285

286

287

288

289

290

291

292

293

294

295

296

297

298

300

301

302

appear when the citrinin issue has been resolved with the help of the current better knowledge of full genomes from industrial strains. Fungi bring a new class of pigments to the food industry, because azaphilones are not present in plants. These azaphilone-producing strains must now be better studied through liquid fermentation of Penicillium/Talaromyces strains, with optimized scale-up. A true fungal anthraquinone alternative to the insect-based carmine is further away from the market, however, due to the nice stability of this vibrant red in foods, and research efforts should be continued and intensified.

\section{References}

Caro Y., Anamale L., Fouillaud M., Laurent P., Petit T., \& Dufossé L. (2012). Natural hydroxyanthraquinoid pigments as potent food grade colorants: an overview. Natural Products and Bioprospecting, 2, 174-193.

Caro Y., Venkatachalam M., Lebeau J., Fouillaud M., Dufossé L. (2016). Pigments and colorants from filamentous fungi. In: “Fungal metabolites.”, Mérillon J.-M. and Ramawat K.G. (Eds.), Springer, Switzerland, ISBN 978-3-319-19456-1, Chapter 26, 170, DOI 10.1007/978-3-319-19456-1_26-1

Ciegler A. (1965). Microbial carotenegenesis. Advances in Applied Microbiology, 7, 1-34.

Ciegler A., Arnold M., and Anderson R.F. (1959). Microbiological production of carotenoids. IV. Effect of various grains on production of $\beta$-carotene by mated strains of Blakeslea trispora. Applied microbiology, 7(2), 94-98.

Dufossé L. (2008). Pigments from microalgae and microorganisms: sources of food colorants. In: Food colorants: chemical and functional properties, $1^{\text {st }}$ edition, Socaciu C. (Ed.), (CRC Press), ISBN 978-0-8493-9357-0, pp. 399-426. 
Dufossé L. (2016). Current and potential natural pigments from microorganisms (bacteria, yeasts, fungi, microalgae). Chapter 16, In: Handbook on natural pigments in food and beverages. Industrial applications for improving food colour, 1st edition, 516 pages, Carle R. and Schweiggert R. (Eds.), (Woodhead Publishing), ISBN 978-0-08-1003718, pp. 337-354.

Dufossé L. (2017a). Pigments, Microbial. In: "Reference Module in Life Sciences", Elsevier Publisher, Oxford, UK, ISBN 978-0-12-809633-8, pp 1-16, DOI 10.1016/B978-0-12809633-8.13091-2

Dufossé L. (2017b). Current carotenoid production using microorganisms. In: Biopigmentation and biotechnological implementations. 1st edition, 277 pages, Singh O.V. (Ed.), (John Wiley \& Sons), ISBN 978-1-119-16614-6, Chapter 4, 87-106.

EFSA (2010). Statement on the divergence between the risk assessment of lycopene by EFSA and the Joint FAO/WHO Expert Committee on Food Additives (JECFA). Scientific opinion from the EFSA Panel on Food Additives and Nutrient Sources added to Food (ANS). EFSA Journal, 8(7):1676 (13 pages).

Fouillaud M., Venkatachalam M., Girard-Valenciennes E., Caro Y., Dufossé L. (2016). Anthraquinones and derivatives from marine-derived fungi: structural diversity and selected biological activities. Marine Drugs, 14(4), 1-64, DOI: 10.3390/md14040064.

Frisvad J.C., Smedsgaard J., Larsen T.O., \& Samson R.A. (2004). Mycotoxins, drugs and other extrolites produced by species in Penicillium subgenus Penicillium. Studies in Mycology, 49, 201-241. 
324 Frisvad J.C., Yilmaz N., Thrane U., Rasmussen K.B., Houbraken J., \& Samson R.A. (2013). Talaromyces atroroseus, a new species efficiently producing industrially relevant red pigments. PLoS One, 19, 8(12):e84102

Fu G., Xu Y., Li Y., \& Tan W. (2007). Construction of a replacement vector to disrupt pksCT gene for the mycotoxin citrinin biosynthesis in Monascus aurantiacus and maintain food red pigment production. Asia Pacific Journal of Clinical Nutrition, 16 Suppl. 1,

Gao J.M., Yang S.X., \& Qin J.C. (2013). Azaphilones: Chemistry and Biology. Chemical Reviews, 113, 4755-4811.

Gessler N.N., Egorova A.S., \& Belozerskaya T.A. (2013). Fungal anthraquinones. Applied Biochemistry and Microbiology, 49, 85-99.

Jonker D., Kuper C.F., Fraile N., Estrella A., and Rodríguez Otero C. (2003). Ninety-day oral

Kumar M., Dwivedi P., Sharma A.K., Sankar M., Patil R.D., \& Singh N.D. (2014). Apoptosis toxicity study of lycopene from Blakeslea trispora in rats. Regulatory Toxicology and Pharmacology, 37, 396-406.

Mantzouridou, F., and Tsimidou, M.Z. (2008). Lycopene formation in Blakeslea trispora. and lipid peroxidation in ochratoxin A- and citrinin-induced nephrotoxicity in rabbits. Chemical aspects of a bioprocess. Trends in Food Science and Technology, 19, 363Toxicology \& Industrial Health, 30, 90-98.

344 Mapari S.A., Thrane U., \& Meyer A.S. (2010). Fungal polyketide azaphilone pigments as future natural food colorants? Trends in Biotechnology, 28, 300-307. 
Mapari S.A.S., Meyer A.S., Thrane U., \& Frisvad J.C. (2009). Identification of potentially safe promising fungal cell factories for the production of polyketide natural food colorants using chemotaxonomic rationale. Microbial Cell Factories, 8, 24.

Mapari S.A.S., Meyer A.S., Thrane U., Frisvad J. (2011). Production of monascus-like pigments. Patent US 20110250656

Mapari S.A.S., Meyer A.S., Thrane U., Frisvad J. (2012). Production of monascus-like pigments. Patent WO2012022765

McNeil B., Archer D., Giavasis I. \& Harvey L. (2013). Microbial production of food ingredients, enzymes and nutraceuticals. Woodhead Publishing, London, 610 pages, ISBN: 978-0-85709-343-1

Milićević D.R., Škrinjar M. \& Baltić T. (2010). Real and perceived risks for mycotoxin contamination in foods and feeds: Challenges for food safety control. Toxins, 2(4): $572-592$.

Morales-Oyervides L., Oliveira J.C., Sousa-Gallagher M.J., Méndez-Zavala A., Montañez J.C. (2015). Effect of heat exposure on the colour intensity of red pigments produced by Penicillium purpurogenum GH2. Journal of Food Engineering, 164, 21-29.

Ogihara, J., Kato, J., Oishi, K., Fujimoto, Y., Eguchi, T., (2000). Production and structural analysis of PP-V, a homologue of monascorubramine, produced by a new isolate of Penicillium sp. Journal of Bioscience and Bioengineering, 90(5), 549-554.

Petruzzi L., Baiano A., De Gianni A., Sinigaglia M., Corbo M.R., Bevilacqua A. (2015). Differential adsorption of ochratoxin A and anthocyanins by inactivated yeasts and 
yeast cell walls during simulation of wine aging. Toxins, 7(10), 4350-65. doi:

Solfrizzo M., Piemontese L., Gambacorta L., Zivoli R., Longobardi F. (2015). Food coloring agents and plant food supplements derived from Vitis vinifera: a new source of human exposure to ochratoxin A. Journal of Agricultural and Food Chemistry 63(13), 3609-

Torres F.A., Zaccarim B.R., de Lencastre Novaes L.C., Jozala A.F., Dos Santos C.A., Teixeira M.F., Santos-Ebinuma V.C. (2016). Natural colorants from filamentous fungi. Applied Microbiology and Biotechnology, 100(6), 2511-2521.

Watson E. (2014). FDA approves use of higher levels of tomato lycopene to colour processed meats, offering alternative to carmine, Red \#40. www.foodnavigatorusa.com/

Woo P.C., Lam C.W., Tam E.W., Lee K.C., Yung K.K., Leung C.K., Sze K.H., Lau S.K., \& Yuen K.Y. (2014). The biosynthetic pathway for a thousand-year-old natural food colorant and citrinin in Penicillium marneffei. Scientific Reports 4, 6728. doi:

Yang Y., Liu B., Du X., Li P., Liang B., Cheng X., Du L., Huang D., Wang L., \& Wang S. (2015). Complete genome sequence and transcriptomics analyses reveal pigment biosynthesis and regulatory mechanisms in an industrial strain, Monascus purpureus YY-1. Scientific Reports, 5, 8331. doi: 10.1038/srep08331 
388 Figure 1. Fungal reds i) already in use in the food industry (carotenoid lycopene from Blakeslea, polyketides from Monascus), ii) at development stage (Monascus-like pigments from Talaromyces species, hydroxy-anthraquinones from many fungi).

TOP LEFT: mycelium (morphological detail) of Blakeslea trispora; chemical formula of the carotenoid lycopene; red-colored foods using fungal lycopene.

393 TOP RIGHT: Monascus purpureus growing on a Petri dish; chemical formula of the polyketide 394 monascorubrin.

BOTTOM LEFT: microscopic view (morphological detail) of Talaromyces sp.; chemical formula 396 of N-threonine monascorubramine.

397 BOTTOM RIGHT: mycelium (morphological detail) of Eurotium cristatum; chemical formula of 398 erythroglaucin; foods which could be colored by the red hydroxy-anthraquinones.

399 (for color view, please refer to the online version of this article).

400 Figure 2. Food-oriented fungal reds that emerged independently at a world level (initial 401 genus/species $^{\mathbf{a}}$ and geographical location).

$402{ }^{a}$ Taxonomic rearrangements have occurred since the first publications about some of the microorganisms. (Please refer to the 403 main text for references of the researches conducted in each country). 

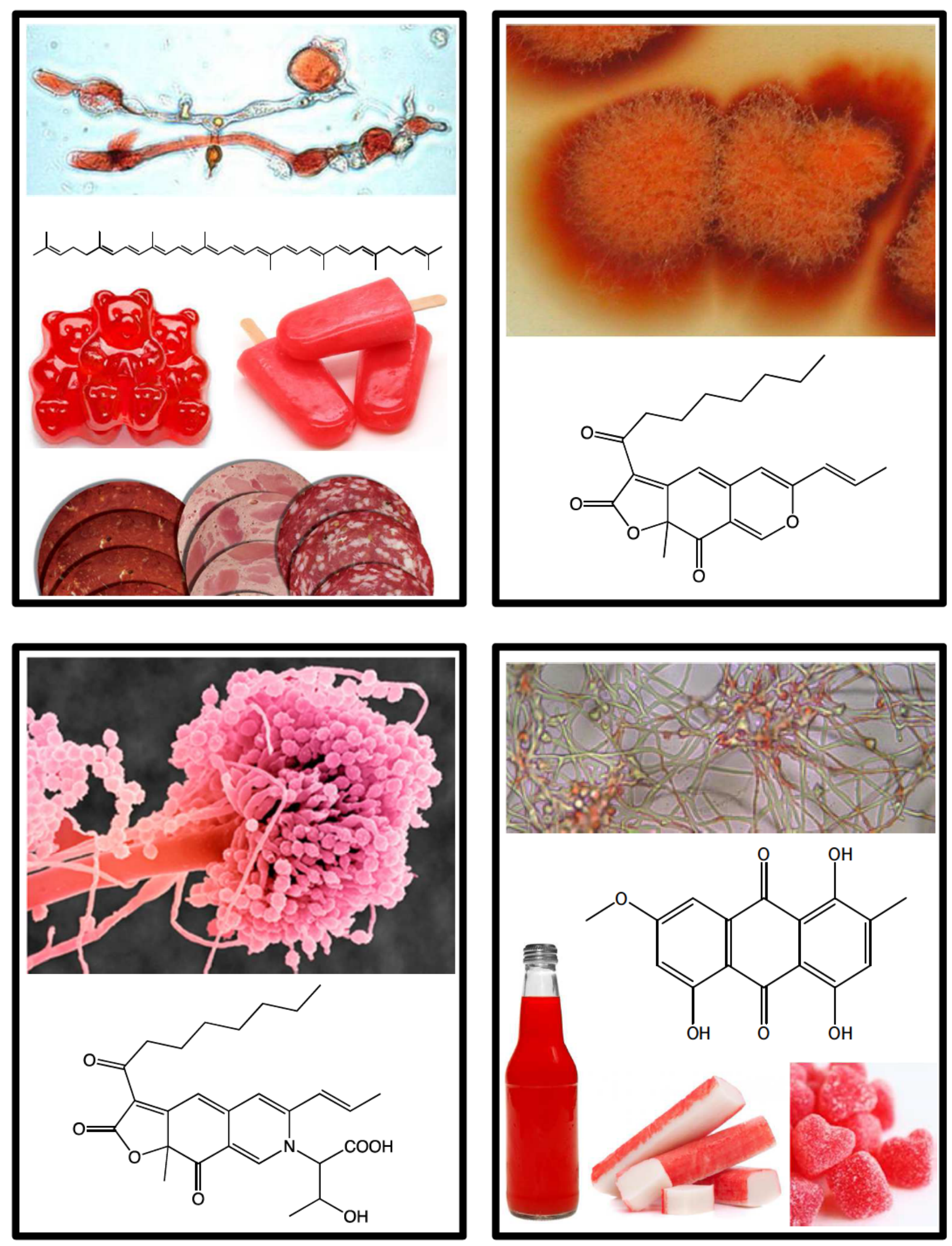


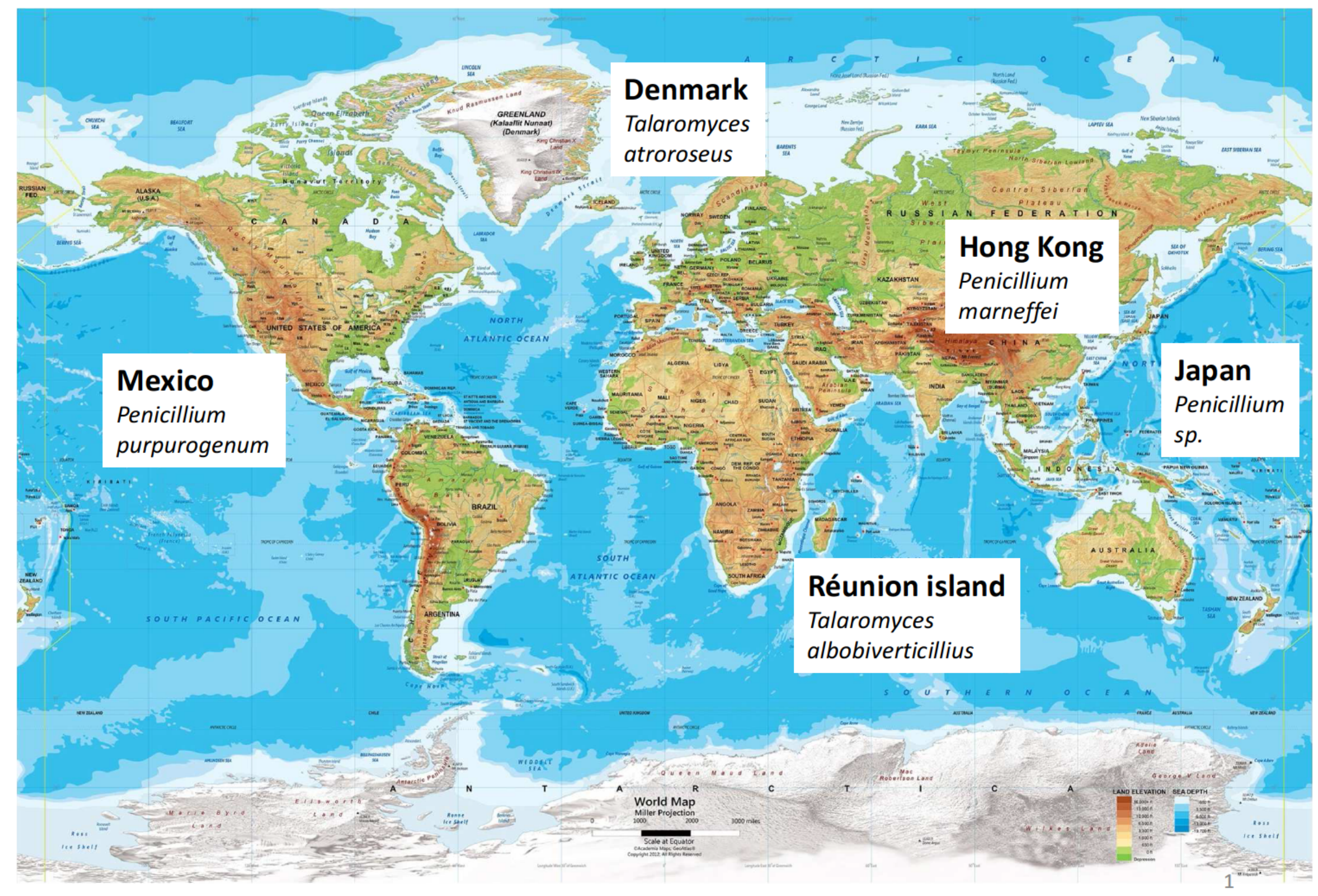

